\title{
Heat generates oxidized linoleic acid metabolites that activate TRPV1 and produce pain in rodents
}

\author{
Amol M. Patwardhan, ${ }^{1}$ Armen N. Akopian, ${ }^{1}$ Nikita B. Ruparel, ${ }^{2}$ Anibal Diogenes, \\ Susan T. Weintraub, ${ }^{3}$ Charis Uhlson, ${ }^{4}$ Robert C. Murphy, ${ }^{4}$ and Kenneth M. Hargreaves ${ }^{1}$ \\ ${ }^{1}$ Department of Endodontics, ${ }^{2}$ Department of Cellular and Structural Biology, and \\ 3Department of Biochemistry, University of Texas Health Science Center at San Antonio, San Antonio, Texas, USA. \\ ${ }^{4}$ Department of Pharmacology, University of Colorado Health Sciences Center, Denver, Colorado, USA.
}

\begin{abstract}
The transient receptor potential vanilloid 1 (TRPV1) channel is the principal detector of noxious heat in the peripheral nervous system. TRPV1 is expressed in many nociceptors and is involved in heat-induced hyperalgesia and thermoregulation. The precise mechanism or mechanisms mediating the thermal sensitivity of TRPV1 are unknown. Here, we have shown that the oxidized linoleic acid metabolites 9- and 13-hydroxyoctadecadienoic acid (9- and 13-HODE) are formed in mouse and rat skin biopsies by exposure to noxious heat. 9- and 13-HODE and their metabolites, 9- and 13-oxoODE, activated TRPV1 and therefore constitute a family of endogenous TRPV1 agonists. Moreover, blocking these substances substantially decreased the heat sensitivity of TRPV1 in rats and mice and reduced nociception. Collectively, our results indicate that HODEs contribute to the heat sensitivity of TRPV1 in rodents. Because oxidized linoleic acid metabolites are released during cell injury, these findings suggest a mechanism for integrating the hyperalgesic and proinflammatory roles of TRPV1 and linoleic acid metabolites and may provide the foundation for investigating new classes of analgesic drugs.
\end{abstract}

\section{Introduction}

The TRP family of ligand-gated ion channels consists of several subgroups, including the vanilloid subfamily (transient receptor potential vanilloid [TRPV]). The first member of the subfamily to be discovered, TRPV1, is an extensively studied channel (1-5) that is expressed in a substantial proportion of pain-sensing sensory neurons, termed nociceptors. TRPV1 can be activated by a variety of endogenous lipids (including lipoxygenase and phospholipase D metabolites of arachidonic acid) and by exogenous substances such as capsaicin (the pungent compound in hot chili peppers) (6). We recently discovered that linoleic acid metabolites are synthesized in the spinal dorsal horn following the afferent barrage due to stimuli such as peripheral inflammation and constitute what we believe to be a novel, physiologically active family of endogenous TRPV1 ligands that mediates central sensitization to mechanical stimuli (7).

In the periphery, TRPV1 also serves as a detector for noxious heat $\left(>43^{\circ} \mathrm{C}\right)(6)$, and pharmacological and gene deletion studies have shown that TRPV1 is important in inflammatory heat hyperalgesia and thermoregulation (8-9). However, the precise mechanism of heat activation of TRPV1 remains unknown. We found that endogenous TRPV1 agonists are formed on exposure of cell membranes to noxious heat. The released compounds activate TRPV1 and contribute to the thermal responsiveness of this channel.

\section{Results}

The hypothesis of heat-evoked generation of endogenous TRPV1 ligand(s) was evaluated by exposing mouse skin biopsies to basal $\left(37^{\circ} \mathrm{C}\right.$, control $)$ or noxious $\left(48^{\circ} \mathrm{C}\right.$, heated $)$ temperatures. Aliquots

Conflict of interest: The University of Texas has claimed intellectual property on this discovery. The authors have declared that no other conflict of interest exists. Citation for this article: J Clin Invest. 2010;120(5):1617-1626. doi:10.1172/JCI41678. of the 2 superfusates were applied at room temperature to cultured trigeminal ganglia (TG) neurons from WT or TRPV1 KO mice. In contrast to control superfusates, the application of superfusates collected from heated skin rapidly increased intracellular calcium $\left(\left[\mathrm{Ca}^{2+}\right]_{\mathrm{i}}\right)$ levels, but only in WT neurons that were also capsaicin sensitive (Figure 1A). Conversely, superfusates collected from heated skin produced no changes in $\left[\mathrm{Ca}^{2+}\right]_{i}$ when applied to neurons from TRPV1 KO animals (Figure 1B; mustard oil [MO] served as a positive control). These results indicate that heated skin releases compound(s) that activate TRPV1 (Figure 1C). Interestingly, the TRPV1-activating property was only observed in superfusates collected from skin biopsies exposed to noxious temperatures $\left(\geq 43^{\circ} \mathrm{C}\right.$; Figure $\left.1 \mathrm{D}\right)$. In addition, samples obtained from heated plastic culture wells (i.e., no skin biopsies) did not alter $\left[\mathrm{Ca}^{2+}\right]_{i}$ in TG neurons, demonstrating that these liberated compounds were of biological origin.

To further characterize the TRPV1 specificity of these endogenous, heat-generated compound(s), we evaluated the effect of pretreatment with the TRPV1 antagonist iodoresiniferatoxin (I-RTX). I-RTX completely blocked the activation of neurons by compound(s) released from heated skin (Figure 1E). In addition, application of these compound(s) triggered prompt increases in $\left[\mathrm{Ca}^{2+}\right]_{\mathrm{i}}$ in CHO cells expressing TRPV1 but not in negative control CHO cells (Figure 1, F and G). Interestingly, the efficacy of TRPV1 activation by these heat-generated compounds was greater in sensory neurons compared with $\mathrm{CHO}$ cells. Heated superfusate may contain other messengers (e.g., PGE2) that sensitize TRPV1 in native cells that endogenously express receptors for these substances (10-11). Moreover, in neurons cultured in the presence of nerve growth factor (NGF), TRPV1 is phosphorylated and in a sensitized state (12). Under whole-cell voltage clamp conditions, the local application of compound(s) isolated from 

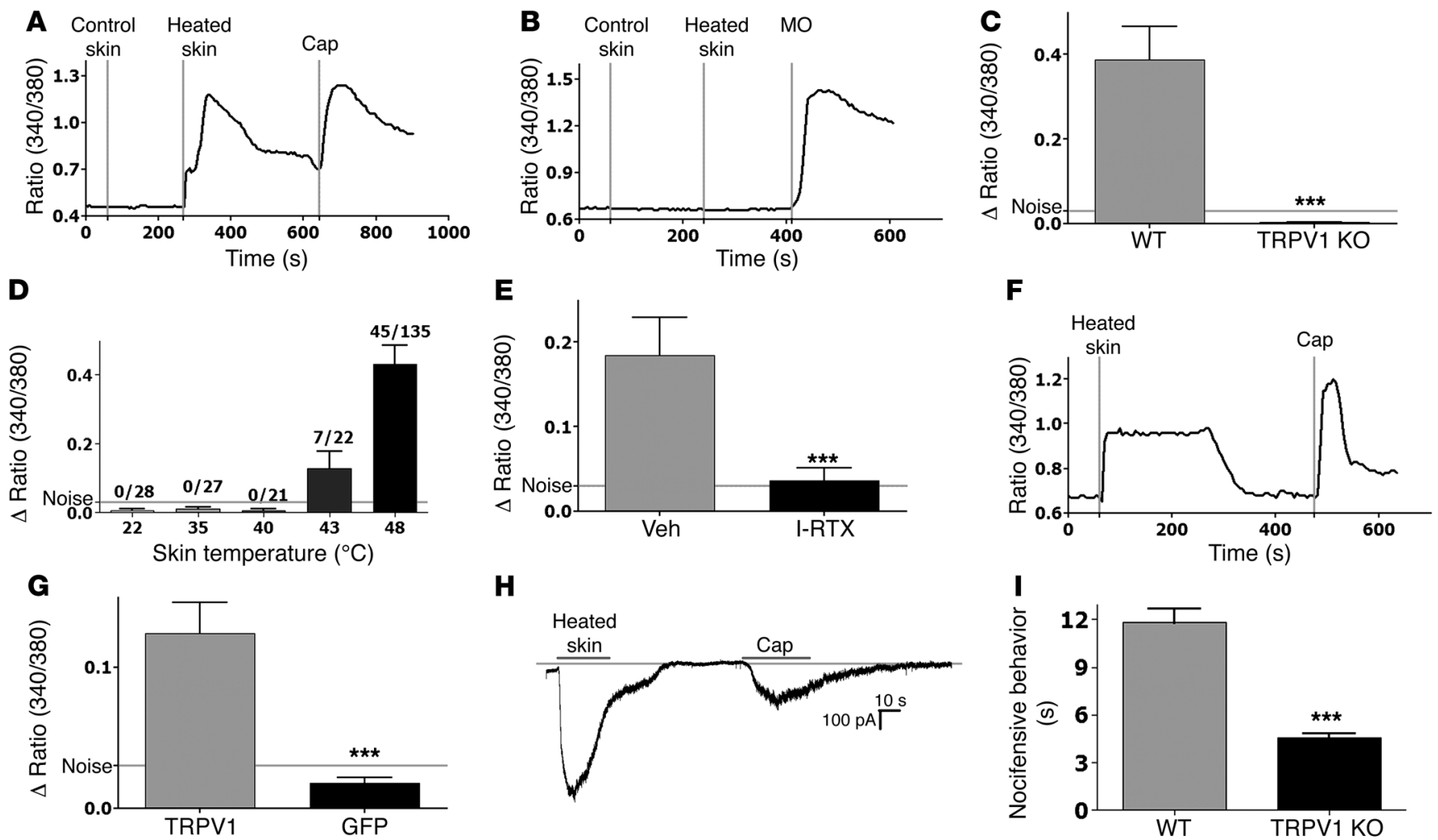

Figure 1

Heated skin evokes endogenous TRPV1 ligand(s). (A) Effect of superfusates, collected from 6 mouse skin biopsies $(1.5 \times 1.5 \mathrm{~cm})$ after exposure to noxious $\left(48^{\circ} \mathrm{C}\right.$ for $\left.20 \mathrm{~min}\right)$ or control temperatures $\left(37^{\circ} \mathrm{C}\right.$ for 20 minutes), to TG neurons from WT mice (positive control: capsaicin [Cap], $100 \mathrm{nM}$ ) using single-cell calcium imaging. Superfusates were applied after cooling to room temperature. Results are plotted as the $340 / 380$ ratio. (B) The effect of same superfusate (1A) on calcium levels in TG neurons from TRPV1 KO mice. Positive control: MO (50 $\mu \mathrm{M})$. (C) Graph summarizing comparison of heated skin superfusate application to WT or TRPV1 KO neurons ( $n=48$ for WT and 36 for TRPV1 KO; $P=0.00001)$. Mean \pm SEM. Results are plotted as $\Delta$ ratio 340/380 ( $\Delta=$ maximum peak 340/380 - baseline 340/380). (D) Temperature dependence of endogenous TRPV1 agonist(s) release (no. of responder/total no. of neurons). (E) Effect of I-RTX (200 nM) pretreatment on superfusate-evoked (mouse skin, heated at $48^{\circ} \mathrm{C}$ for 20 minutes) responses in TG neurons from WT mice ( $n=44$ for vehicle [Veh], 31 for I-RTX; $\left.P=0.0003\right)$. (F) Heated skin superfusate applied to TRPV1 CHO cells. Positive control: capsaicin (100 nM). (G) Graph summarizing comparison of heated skin superfusate effect on CHO cells expressing TRPV1 ( $n=56$ for TRPV1, 43 for GFP, negative control; $P=0.00001$ ). (H) Representative inward current in rat TG neuron by heated mouse skin superfusate and capsaicin. (I) Nocifensive behavior (WT vs. TRPV1 KO mice) evoked by hind paw injection of compound(s) isolated from previously heated skin biopsies $(n=5-6$ per group; $P=0.0003)$. ${ }^{* * *} P<0.001$.

heated skin superfusate evoked a profound inward current (902 $\pm 203 \mathrm{pA}, n=6$ cells) that was restricted to the capsaicin-sensitive subpopulation of rat TG neurons (Figure $1 \mathrm{H}$ ). Finally, the heat-evoked components isolated from a $\mathrm{C}_{18}$ reversed-phase column produced significantly greater nocifensive behavior when injected intraplantar (ipl) into the hind paws of WT mice compared with TRPV1 KO mice (Figure 1I).

The heat-evoked generation of endogenous TRPV1 ligand(s) was not restricted to mouse skin tissue. The application of noxious heat to nontransfected $\mathrm{CHO}$ cells, rat skin biopsies, or even skin biopsies from TRPV1 KO mice all resulted in the release of compounds that rapidly activated TRPV1 (Supplemental Figure 1; supplemental material available online with this article; doi:10.1172/JCI41678DS1). These results suggest that the machinery required for the generation of endogenous TRPV1 ligand(s) is widely expressed and does not require the presence of TRPV1 for the release of these compounds.

We next sought to identify the endogenous TRPV1 ligand(s). The only mass spectrometrically measured ion that appeared to be unique for the TRPV1-activating HPLC fraction appeared at $m / z 295$ (Figure 2A). In some control samples from nonheated skin, there was a detectable ion at $m / z 295$ in the aliquot, but the abundance of this ion was always less than that found in aliquots from heated skin. To identify the component that generated this negative ion, we carried out collisional activation of the $m / z 295$ ion from a biologically active HPLC fraction (fraction 22). The observed product ions (Figure $2 \mathrm{~B}$ ) were consistent with 2 oxidized metabolites of octadecadienoic acid, namely 9-hydroxyoctadecadienoic acid (9-HODE) and 13-HODE (13). Linoleic acid is one of the most abundant fatty acids in the body and is a major component of the plasma membrane of nearly all cells. Under conditions such as inflammation, cytotoxicity, or hypotension, linoleic acid is rapidly oxidized to form biologically active metabolites such as 9-HODE and 13-HODE (14-16). Both 9-HODE and 13-HODE were released in increasing amounts from mouse skin biopsies exposed to a noxious temperature range (Figure 2, C and D). Interestingly, the temperature dependence curve for HODE generation was strikingly 

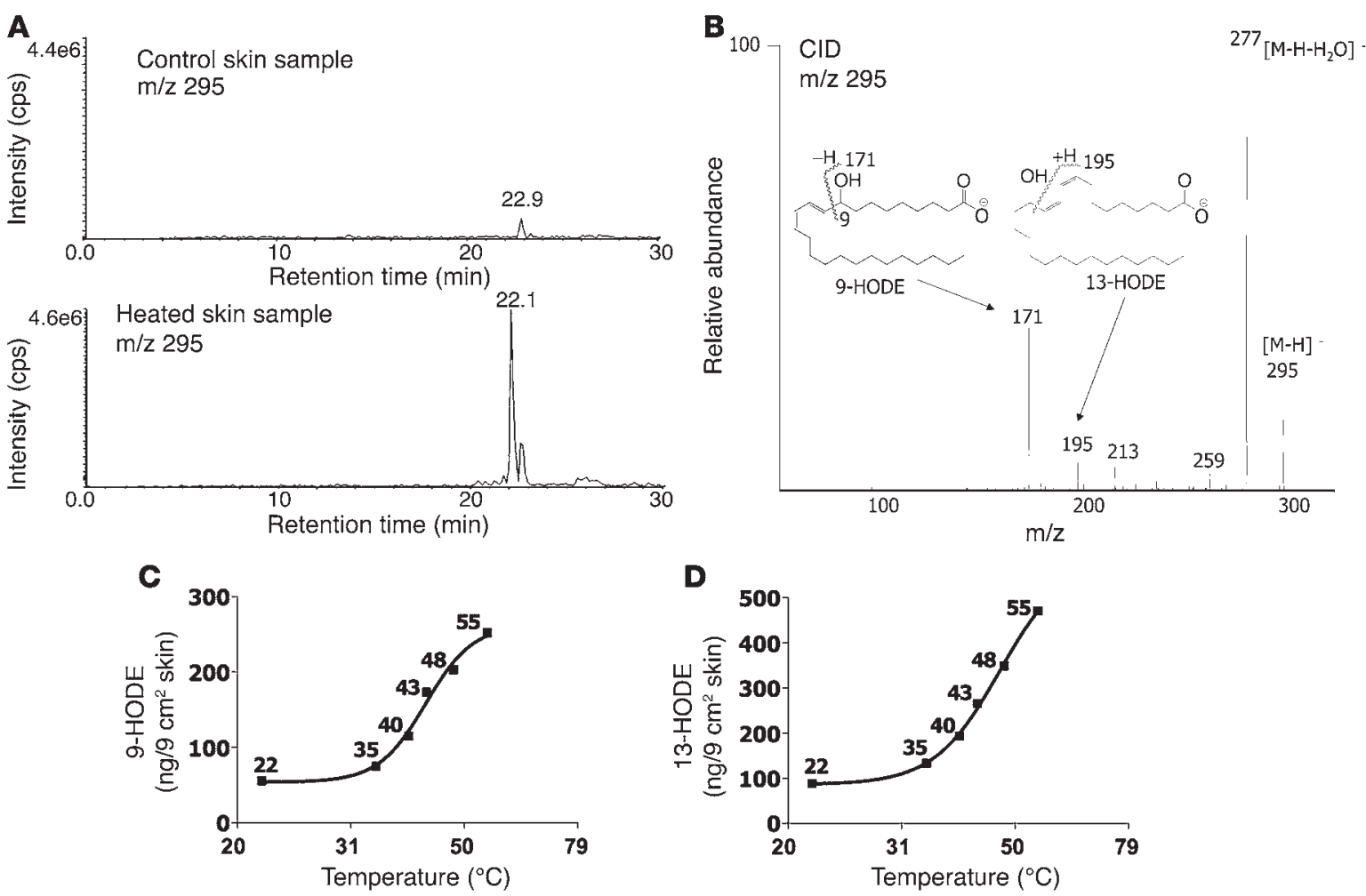

Figure 2

Heating increases oxidized linoleic acid metabolites in skin. (A) HPLC comparison of unique substances in superfusates collected from mouse skin biopsies $(1.5 \times 1.5 \mathrm{~cm})$ after exposure to noxious heat $\left(48^{\circ} \mathrm{C}\right.$ for $\left.20 \mathrm{~min}\right)$ or control temperatures $\left(37^{\circ} \mathrm{C}\right.$ for $\left.20 \mathrm{~min}\right)$. The TRPV 1 activity of each fraction was evaluated using calcium imaging of TG neurons cultured from rats and CHO cells expressing TRPV1 (data not shown). cps, counts per second. (B) Evaluation of product ions formed from HPLC fraction 22 following collisional activation of the [M-H]- ion at $m / z 295$ with tandem quadrupole mass spectrometer monitoring of either $\mathrm{m} / \mathrm{z} 171$ (9-HODE) or $\mathrm{m} / \mathrm{z} 195$ (13-HODE). (C and D) Temperature-dependent release of 9-HODE (C) and 13-HODE (D) into mouse skin superfusates collected after 20 minutes exposure to a given temperature. 9- and 13-HODE were detected by HPLC/MS as described ( $x$ axis has a logarithmic scale).

similar to that observed with heat activation of TRPV1, with more shallow slope at lower temperatures and steep slope at noxious temperature (17).

We then characterized the pharmacological activity of synthetic 9-HODE and related compounds. The application of 9-HODE activated WT TG neurons (Figure 3A) but not TRPV1 KO neurons (Figure 3B). The neuronal population that responded to 9-HODE in WT mice completely coincided with the capsaicin-sensitive population (75/75 neurons). The application of 9-HODE to neurons cultured from TRPV1 KO mice resulted in a slight response in $4 \%$ of tested neurons (3/66); however, both the magnitude and time course of $\left[\mathrm{Ca}^{2+}\right]_{\mathrm{i}}$ accumulation were substantially reduced (Figure $3 \mathrm{C}$ ). In a whole-cell voltage-clamp recording, administration of 9-HODE $(100 \mu \mathrm{M})$ evoked an inward current $(2286 \pm 455 \mathrm{pA}, n=6$; Figure 3D) in neurons that also responded to capsaicin. The application of 9-HODE to cultured rat TG neurons triggered the release of immunoreactive calcitonin gene-related peptide (iCGRP), with effects evident at $10 \mathrm{nM}$ and a half-maximal effective concentration $\left(\mathrm{EC}_{50}\right)$ of approximately $300 \mathrm{nM}$ (Figure 3E). Similarly, in CHO cells expressing TRPV1, 9-HODE was able to evoke calcium accumulation at concentrations as low as $10 \mathrm{nM}$ (data not shown). This concentration range encompasses levels found in the heated skin superfusate in the mass spectrometry (MS) analysis. 13-HODE generated inward currents in CHO cells transfected with TRPV1, with an $\mathrm{EC}_{50}$ of approximately $800 \mathrm{nM}$ (Figure 3F). Application of 9-oxoODE, a metabolite of 9-HODE, also increased $\left[\mathrm{Ca}^{2+}\right]_{\mathrm{i}}$ accumulation (Figure 3G). Another major metabolic pathway of linoleic acid leads to formation of 13-HODE and its metabolite, 13-oxoODE. The relative efficacy of 9-HODE, 13-HODE, 9-oxoODE, and 13-oxoODE for evoked $\left[\mathrm{Ca}^{2+}\right]_{\mathrm{i}}$ levels was evident when tested in cultured neurons from WT mice (Figure 3H) and in TRPV1expressing CHO cells (Figure 3I). Both 9-HODE and 13-HODE were inactive in CHO cells expressing TRPV2, -V3, or -V4 (data not shown). In addition, the ipl hind paw injection of 9-HODE in rats produced a dose-related increase in spontaneous nocifensive behavior that was significantly increased when 9-HODE was coinjected with a mixture of 13-HODE, 9-oxoODE, and 13-oxoODE (Figure 4A). In addition to inducing acute pain in rats, the ipl injection of 9-HODE in mice produced thermal hyperalgesia in WT but not TRPV1 KO animals (Figure 4B).

If heat-generated linoleic acid metabolites contribute to the heat sensitivity of TRPV1, then these compounds should remain active in TRPV1 mutants that are heat sensitive but capsaicin insensitive. We transfected CHO cells with $\operatorname{Trp} v 1^{511}$ and $\operatorname{Tr} p v 1^{512}$, 2 mutants that are responsive to heat and $\mathrm{pH}$, but not capsaicin (18). Application of either the endogenous compounds isolated from heated skin or synthetic $9-\mathrm{HODE}$, increased $\left[\mathrm{Ca}^{2+}\right]_{\mathrm{i}}$ in these Trpv1 mutant-transfected CHO cells (Supplemental Figure 2), 
A

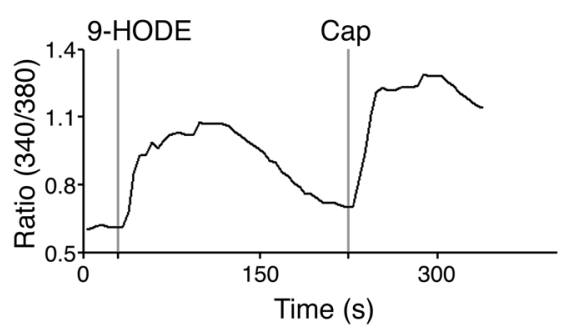

D

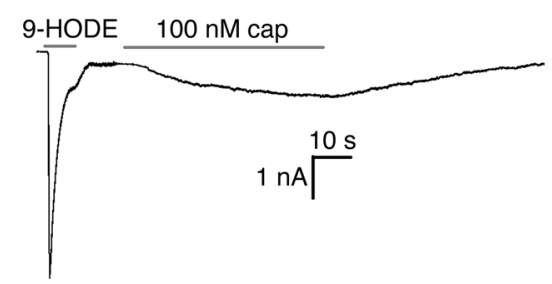

G

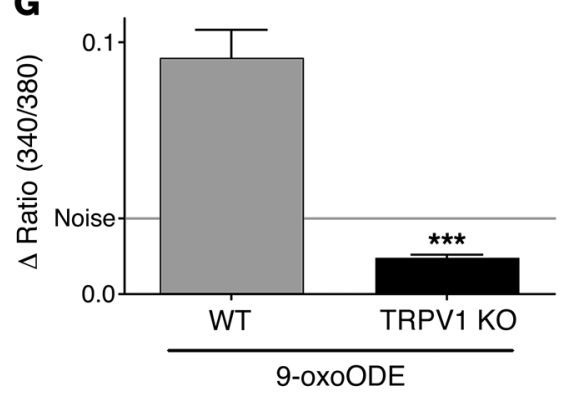

B

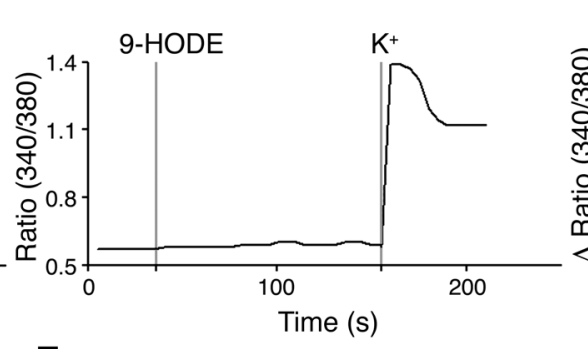

E

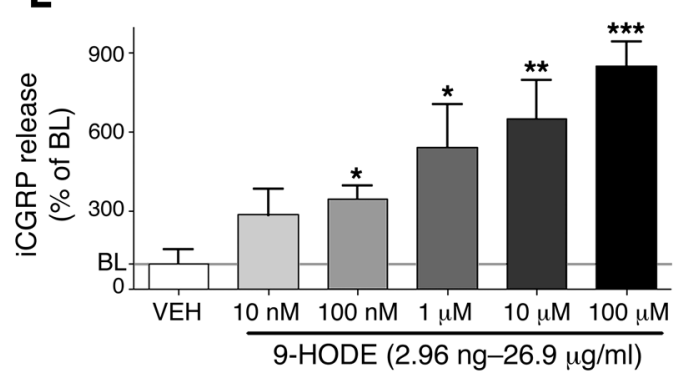

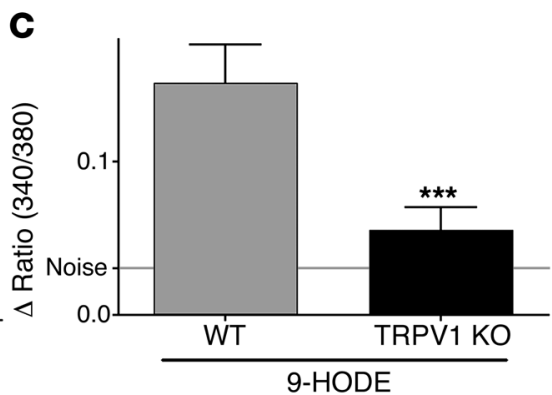

$\mathbf{F}$

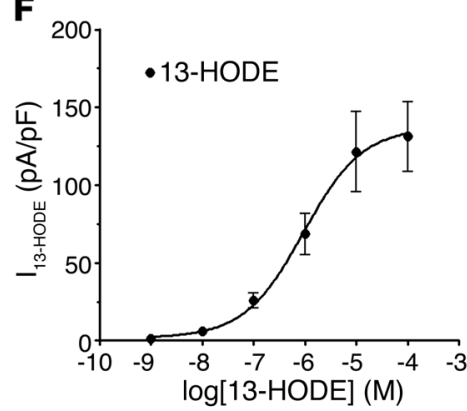

H

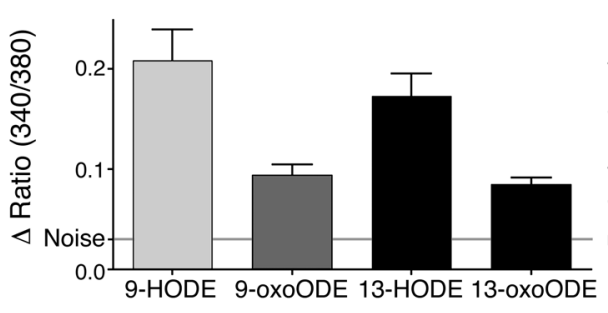

I

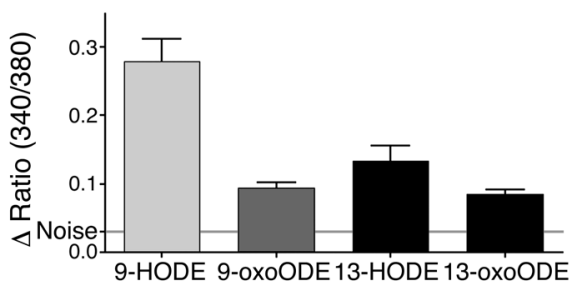

Figure 3

Oxidized linoleic acid metabolites are TRPV1 agonists. (A and B) Synthetic 9-HODE (100 $\mu \mathrm{M})$ activates TG neurons from WT mice but not from TRPV1 KO mice as measured using calcium imaging. (C) Graph summarizing comparison of 9-HODE activation of TG neurons from WT versus TRPV1 KO mice ( $n=50$ for WT and 66 for TRPV1 KO; $P=0.0002)$. (D) Whole-cell recording demonstrates activation of a rat TG neuron by synthetic 9-HODE $(100 \mu \mathrm{M})$ and capsaicin. (E) Concentration-response effects of applying synthetic 9-HODE (15 minutes) on iCGRP release from cultured rat TG neurons as measured by radioimmunoassay $(n=8-16$ wells/group; $P=0.0001)$. BL, baseline. (F) Concentration-response curve for synthetic 13-HODE. Responses (pA/pF) were recorded from TRPV1-expressing CHO cells. (G) Effect of applying synthetic 9-oxoODE (100 $\mu \mathrm{M})$ on TG neurons from WT mice ( $n=71$ for WT and 83 for TRPV1 KO; $P=0.0007)$. ${ }^{*} P<0.05 ;{ }^{* *} P<0.01$; ${ }^{* *} P<0.001$. (H and I) Comparison of the effects of applying linoleic acid metabolites (all $100 \mu \mathrm{M} ; n=47-75$ ) to TG neurons cultured from WT mice (H) or to CHO cells transfected with TRPV1 (I) as measured by calcium imaging.

although the magnitude was somewhat lower than WT TRPV1. AMG8562 is a TRPV1 antagonist that does not block heat activation of TRPV1 but is a highly potent and effective blocker of capsaicin activation of TRPV1 (19). Pretreatment with a saturating concentration of AMG8562 had no effect on 9-HODE-evoked calcium accumulation in rat TG neurons but completely blocked the effects of capsaicin (Supplemental Figure 2). Collectively, these data indicate that an endogenous compound or compounds isolated from heated skin and synthetic 9-HODE activate TRPV1 in a manner that is similar to noxious heat but different from that of capsaicin. The data suggest that the ligand recognition site on TRPV1 for these substances is distinct from the capsaicin site, with at most only a partial overlap.

Next, we evaluated whether heat-evoked activation of rat TG neurons is inhibited by interventions directed against linoleic acid metabolites. Since oxidized linoleic acid metabolites can be formed either enzymatically (via lipoxygenase) or spontaneous- ly (via free radicals; ref. 15), we used nordihydroguaiaretic acid (NDGA), since this compound inhibits both pathways $(20,21)$. Pretreatment with NDGA produced approximately $50 \%$ reduction in $\mathrm{I}_{\text {heat }}$ (Figure 5 , A and B). In addition, heat-evoked $\left(48^{\circ} \mathrm{C}\right)$ iCGRP release was significantly inhibited by pretreatment with NDGA and I-RTX but not by indomethacin, a cyclooxygenase inhibitor (Figure 5C). Thus, the heat-evoked release of endogenous compounds is independent of a cyclooxygenase pathway. The effect of NDGA was concentration dependent in both assays (electrophysiology and CGRP release), with a statistically insignificant effect at $1 \mu \mathrm{M}$ and minimal inhibition $(\sim 23 \%)$ at $10 \mu \mathrm{M}$. Higher concentrations of NDGA $(>100 \mu \mathrm{M})$ evoked inward current and iCGRP release, limiting its use to evaluate the effect on heat activation of neurons. We then directly evaluated whether intracellular immunoneutralization of 9-HODE and 13-HODE alters the responsiveness of sensory neurons to noxious heat. The pipette solution was loaded with both anti-9-HODE and anti- 

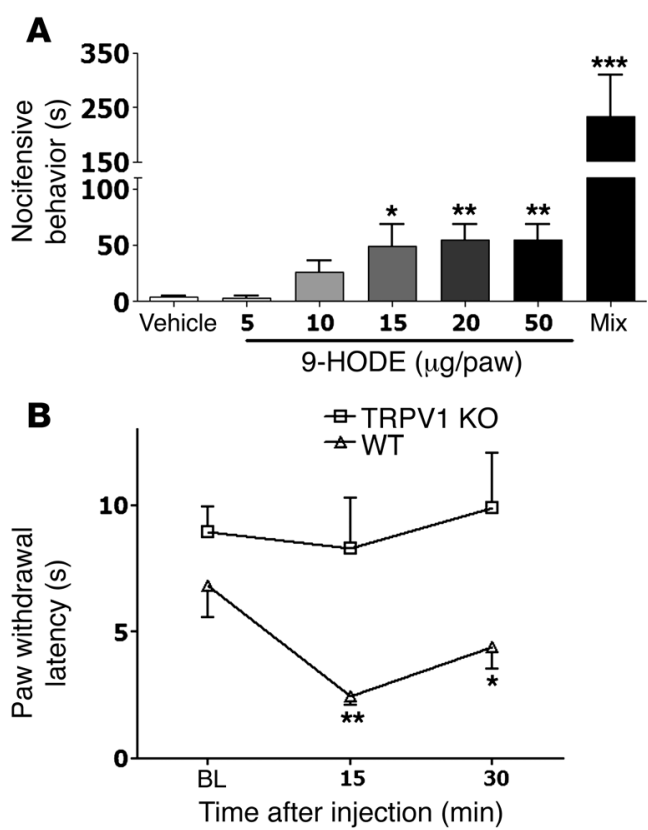

Figure 4

Oxidized linoleic acid metabolites trigger acute pain and thermal hyperalgesia. (A) Effects of an ipl hind paw injection in rats of 9-HODE or a mixture of 9-HODE, 13-HODE, 9-oxoODE, and 13-oxoODE (mix, $25 \mu \mathrm{g}$ each) on spontaneous nocifensive behavior (duration of flinches; $n=4-6 /$ group; ${ }^{\star} P<0.05 ;{ }^{*} P=0.003$ vs. vehicle; ${ }^{* * *} P=0.0001$ vs. all other groups). Observers were blinded to treatment allocation. (B) Effects of an ipl injection of 9-HODE in WT or TRPV KO mice on paw withdrawal latency to a beam of radiant heat $(n=6$ per group; $P=0.007$ at 15 minutes).

13-HODE antibodies and delivered intracellularly via dialysis. This treatment combination decreased $\mathrm{I}_{\text {heat }}$ in a dose-dependent fashion, with the greatest dose achieving a 54\% reduction in $\mathrm{I}_{\text {heat }}$ $(P<0.01$; Figure 5, D and E). Interestingly, dialysis of either antibody alone did not significantly reduce $\mathrm{I}_{\text {heat, }}$, implicating a redundancy in the signaling properties of these compounds. Moreover, both antibodies when used alone demonstrated a statistically insignificant trend toward sensitization of $\mathrm{I}_{\text {heat }}$ (increase of $17 \%$, $P=0.17)$. Importantly, dialysis with even the highest dose of the antibody combination did not affect capsaicin-evoked (100 nM) inward currents (vehicle control: $1.34 \pm 0.4 \mathrm{nA}$ vs. antibodies: $1.53 \pm 0.3 \mathrm{nA} ; n=6$ cells each), suggesting that the inhibitory effect of the antibodies was specific to heat activation of TRPV1. Control experiments demonstrated that intracellular delivery of NDGA or the anti-HODE antibodies had no effect on the responses by extracellular bath delivery of 9-HODE, suggesting that the reservoir of 9-HODE in the bath can saturate available intracellular antibodies (Supplemental Figure 3). Finally, we evaluated the behavioral consequences of blockade of linoleic acid oxidation and neutralization of HODEs on thermal nociception in rats. The ipl hind paw injection of NDGA evoked significant thermal antinociception compared with the contralateral paw of the same animals or with vehicle-injected paws in a separate set of animals (Figure 5F). Moreover, the ipl injection of a combination of antibodies against 9- and 13-HODE also evoked significant antinociception in the injected paw without any effect on the contralateral paw (Figure 5G). Demonstration of an effect restricted to the ipsilateral (injected) hind paw indicates that these drugs produced their antinociceptive effect by a peripheral mechanism of action localized in the injected tissue. Collectively, these data indicate that compounds that block the synthesis or actions of linoleic acid metabolites significantly reduce TRPV1 activities in vitro and behavioral responses to heat in vivo.

Next, we compared the latency of TRPV1 activation by heat and by oxidized linoleic acid metabolites (Figure 6). Control studies first determined the inherent latencies resulting from the perfusion system (potassium as positive control; Figure 6B) and delivery of thermal stimuli $\left(48^{\circ} \mathrm{C}\right.$ as positive control; Figure $\left.6 \mathrm{C}\right)$, allowing us to calculate the latencies resulting from application of 9-HODE and to $43^{\circ} \mathrm{C}$ heat. The observed mean latencies to 9-HODE was quite similar to that of $43^{\circ} \mathrm{C}(10-13 \mathrm{~ms}$; Figure $6 \mathrm{~A})$. It should be noted that our latency-measurement equipment had a standard deviation of approximately $5 \mathrm{~ms}$. Under these conditions, there was no significant difference $(P>0.05)$ between the latencies of 9-HODE and heat activation of TRPV1.

We next evaluated the direct activity of linoleic acid on TG neurons and $\mathrm{CHO}$ cells using single-channel recording and calcium imaging (Figure 7). The addition of high concentrations of linoleic acid produced a concentration-dependent increase in TRPV1 activity/open probability $\left(P_{o}\right)$ (Figure 7, A and B). Furthermore, pretreatment with NDGA $(30 \mu \mathrm{M}, 15$ minutes) blocked the effects of even the largest concentrations $(1 \mathrm{mM})$ of linoleic acid on calcium accumulation (340/380 ratios: vehicle/linoleic acid, $0.092 \pm 0.008, n=53$, versus NDGA/linoleic acid $0.032 \pm 0.004$, $n=58, P<0.001$; Figure $7 \mathrm{C})$.

Washing inside-out patches for at least 3 minutes led to the rapid loss of heat activation of TRPV1 (Figure 8). In contrast, application of the TRPV1 ligands 9-HODE (Figure 6A) and capsaicin (Figure 8D) activated TRPV1 even after a 5- to 10-minute washing period, indicating that the lost response to heat was not a result of a loss of TRPV1 responsiveness. The addition of linoleic acid to the heated solution (at a concentration that did not generate responses at room temperature, $22-24^{\circ} \mathrm{C}$ ) resulted in a reappearance of the heat response, indicating that linoleic acid rescued the heat response under these conditions (Figure 8A). Furthermore, linoleic acid did not sensitize the TRPV1 channel, as application of linoleic acid and capsaicin did not further increase the TRPV1 $P_{o}$ (Figure $8 \mathrm{D}$ ). These results can be interpreted to indicate that the addition of linoleic acid provides the necessary precursor for formation of HODEs and that generation of HODEs contributes to restoration of heat sensitivity of TRPV1.

\section{Discussion}

Here, we evaluated the hypothesis that the heat sensitivity of TRPV1 is regulated by the generation of endogenous ligands. We found that heat-generated linoleic acid metabolites comprise a family of physiologically relevant TRPV1 agonists that contribute to the heat responsiveness of this channel. Moreover, our results also suggest a previously unknown mechanism by which TRPV1 might mediate biological actions of oxidized linoleic acid metabolites in conditions such as inflammation and hypotension.

Heat activation of TRPV1 appears to occur in a membranedelimited fashion (during short periods) (4) that is dependent on its $C$ terminus (1) or voltage gating (5). Notably, TRPV1 heat sensitivity is mechanistically distinct from capsaicin sensitivity $(18,19)$. On the basis of these studies and our own results, we propose that heating leads to the generation of oxidized linoleic 
A

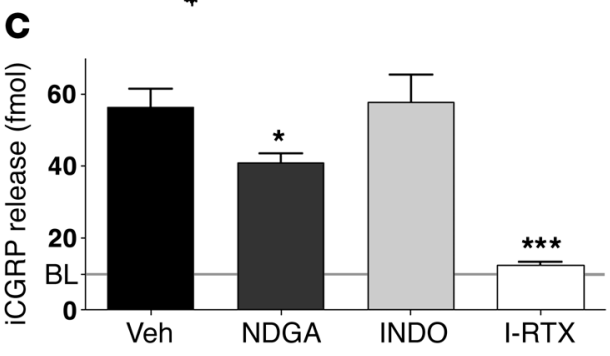

E
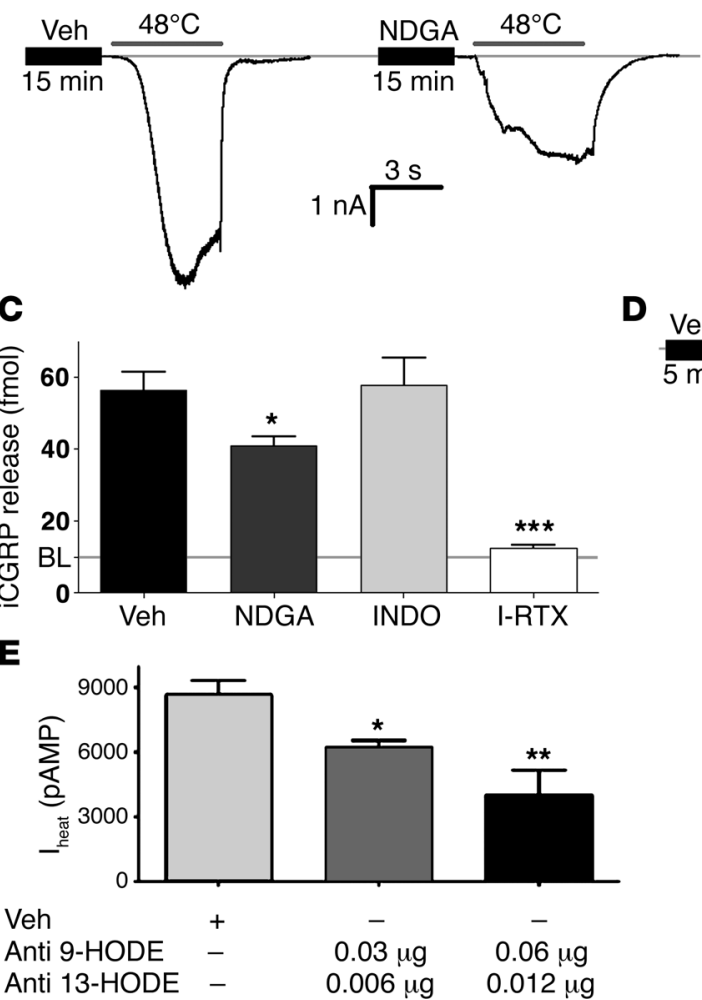

B

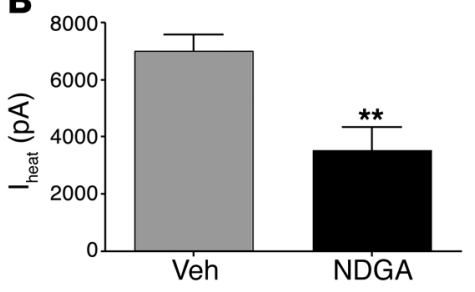

D $\mathrm{Ve}$

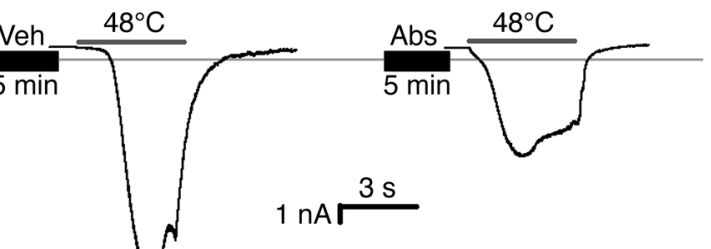

$1 \mathrm{nA} \overbrace{}^{3 \mathrm{~s}}$

$\mathbf{F}$

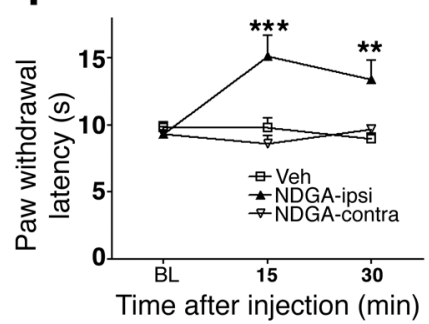

G

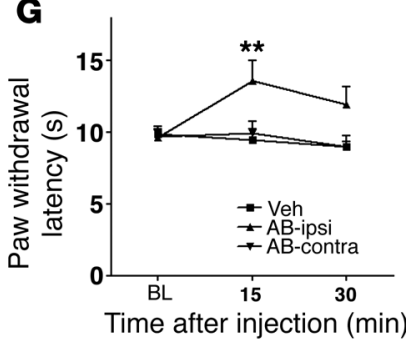

Figure 5

Inhibition of oxidized linoleic acid metabolites decreases heat sensitivity of TRPV1. (A) Effect of applying vehicle or NDGA (30 $\mu$ M, 15 minutes) on whole-cell heat-evoked inward current $\left(I_{\text {heat }}\right)$ in a cultured rat TG neuron. (B) Summary graph shows the effects of NDGA on $I_{\text {heat }}$ in rat TG neurons $(n=19$ for vehicle, 9 for NDGA; $P=0.0018)$. (C) The effect of pre- and cotreatment with NDGA $(10 \mu \mathrm{M})$, indomethacin (INDO) $(2 \mu \mathrm{M})$, and I-RTX (200 nM) on heat-evoked iCGRP release from cultured rat TG neurons $(n=8-16$ wells/group; $P=0.02$ for NDGA and 0.00001 for I-RTX). (D) Effect of an intracellular dialysis (for 5-10 minutes) of either vehicle or a combination of anti-9-HODE and anti-13-HODE antibodies $(0.06$ and $0.012 \mu \mathrm{g}$, respectively) on Ineat in cultured rat TG neurons. (E) Graph summarizing effects of dialysis with antibodies against 9- and 13-HODE on $I_{\text {heat }}$ in cultured rat TG neurons $(n=15$ for vehicle and 7 for each antibody mix group, $P=0.007$; antibody dose refers to amount in a recording pipette). (F and $\mathbf{G})$ Effect of ipl hind paw injection of vehicle, NDGA (F, $n=6 /$ group; $P=0001)$, or a mixture of anti-9-HODE and anti-13-HODE antibodies (G, $25 \mu \mathrm{g}$ each/paw, $n=6$ /group; $P=0.005)$ on paw withdrawal latencies to a beam of radiant heat in rats. Observers were blinded to treatment allocation. ${ }^{*} P<0.05 ;{ }^{* *} P<0.01 ;{ }^{* *} P<0.001$.

acid products in the plasma membrane that are important for TRPV1 responses to noxious thermal stimuli. Since these substances are formed in the membrane in the vicinity of TRPV1, substantially high concentrations of these substances could occur in the local microenvironment. It should be noted that in inflammatory diseases, relatively high levels of HODEs are observed even in extracellular compartments (22-23). Our data indicate that 9-HODE and 13-HODE substantially contribute to the heat responsiveness of TRPV1 in vitro and in vivo. Other factors contributing to the heat responsiveness of TRPV1 could include either the intrinsic heat sensitivity of TRPV1, additional endogenous ligands, or both. Given the temporal resolution of our latency experiments (Figure 6), it is possible that heat directly activates TRPV1, with a subsequent generation of endogenous ligands that further amplifies the heat response. Although other substances may be present in the superfusate, the demonstration that the observed biological actions occur only in WT neurons and not neurons from TRPV1 KO animals (Figure 1C), that pretreatment with I-RTX, a TRVP1 antagonist, blocks the response (Figure 1E), and that superfusate-evoked nocifensive behavior is almost absent in TRPV1 KO animals (Figure 1I) indicate that dominant biological effects can only be attributed to TRPV1 activation. In addition, the in vitro and in vivo results indicate that blockade of the endogenous linoleic acid metabolites substantially decreased responses to thermal stimuli. Notably, the heat sensitivity of another member of the TRP family, TRPV4, is mediated via generation of a soluble ligand (24).

Heated skin releases 9-HODE and 13-HODE and the application of 9-HODE and 13-HODE as well as of their respective metabolites (9- and 13-oxoODE) effectively activates TRPV1. Thus, the net effect of the heated skin superfusate may be attributed to the action of several endogenous lipids on TRPV1; indeed, hind paw injection of 9-HODE has no effect on thermal allodynia in TRPV1 KO animals (Figure 4B). In addition, there was a substantial increase in spontaneous nocifensive behavior when all 4 substances were injected together (Figure 4A). This suggests that the interaction among these linoleic acid metabolites may produce a super-additive effect on TRPV1 activation and nocifensive behaviors. Indeed, such an entourage effect has been proposed for other bioactive lipid families (25-26). The HODEs in the superfusate 
A
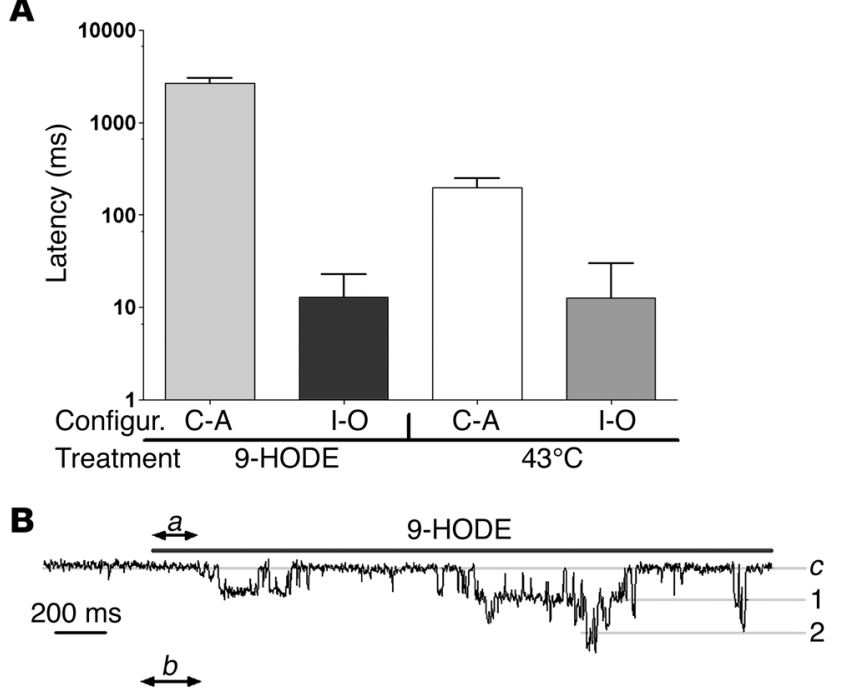

K-solution

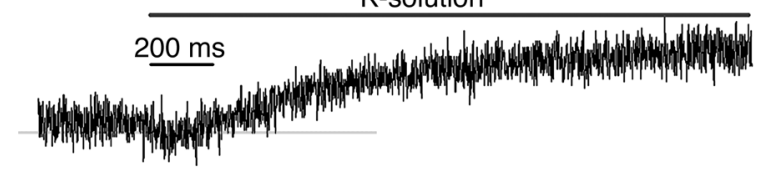

C
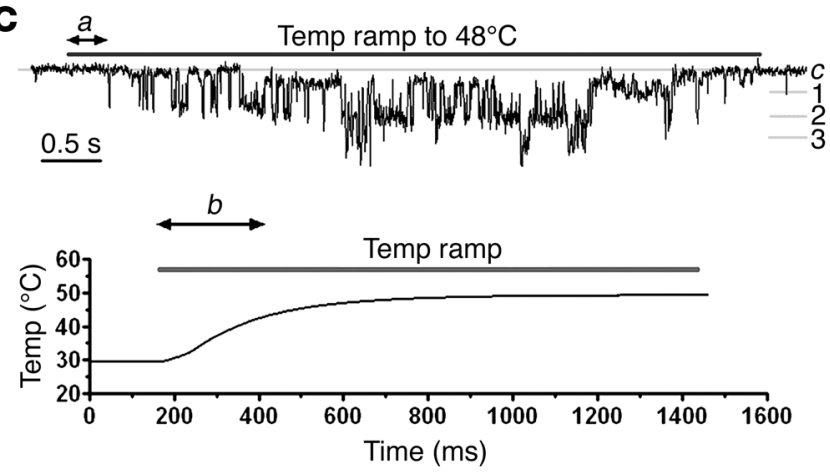

can be generated from both neuronal and nonneuronal tissues $(15,27)$ (Figure 1 and Supplemental Figure 1) and thus may act as both intracellular and intercellular mediators. Intracellular concentrations of these substances in the vicinity of TRPV1 could easily become very high, as they are formed near the plasma membrane. In the thermal nociception test, ipl injection of antibodies to 9- and 13-HODE evoked substantial thermal antinociception. It is unlikely that these antibodies crossed the cell membrane of the sensory terminals and neutralized the intracellular HODEs, suggesting that the intercellular component is important for basal thermal detection. $\mathrm{PGE}_{2}$ was recently proposed as another possible intercellular messenger (10).

Our data demonstrate, for what we believe is the first time, that oxidized linoleic acid metabolites constitute a family of endogenous TRPV1 ligands that are released under physiological conditions in the periphery. The activation of TRPV1 may explain the role of these metabolites in inflammatory or degenerative diseases such as arthritis, atherosclerosis, and psoriasis $(22-23,28)$. In rat and mouse TG neurons, the excitatory effect of 9-HODE and 9-oxoODE was almost entirely dependent on the presence of TRPV1. Thus, it is conceivable that oxidized linoleic acid metabolites may be formed by heat or even other stimuli

\section{Figure 6}

Application of $9-\mathrm{HODE}$ and heat $\left(43^{\circ} \mathrm{C}\right)$ demonstrate similar latencies for activation of TRPV1. (A) The latencies for generation of singlechannel inward currents due to application of either 9-HODE $(100 \mu \mathrm{M})$ or $43^{\circ} \mathrm{C}$ to TRPV1-expressing $\mathrm{CHO}$ cells. $n=6-10$. Recording was carried out in cell-attached (C-A) and inside-out (I-O) configurations (configur.). (B) Representative single-channel inside-out traces illustrating the measurement of the latency for 9-HODE. Latency was calculated as the difference between $a$ and $b$. A solution containing potassium $(50 \mathrm{mM})$ was applied via the recording pipette to measure the inherent latency of the perfusion system $(b)$. Inside-out configuration was established for $3-5$ minutes. $c$ is a close state. 1,2 , and 3 are numbers of channels in the patches. (C) Representative single-channel inside-out traces illustrating the measurement of the latency for $43^{\circ} \mathrm{C}$. Temperature was applied immediately (within 1 minute) after establishment of the inside-out configuration. Latency was calculated as $a-b$, where $b$ is the time to reach $43^{\circ} \mathrm{C}$ in the vicinity of the cells. Temperature ramp was recorded by placing a thermistor in the position of previously recorded cells.

(29) and contribute to ongoing pain or hyperalgesia in inflammatory diseases. This hypothesis suggests that agents blocking either the production or action of these substances could lead to pharmacological interventions for many inflammatory diseases or pain disorders. Indeed, our recent studies demonstrate efficacy of such an approach in blocking TRPV1-mediated central sensitization in the spinal cord (7).

Previous studies have demonstrated that leukotrienes activate TRPV1, epoxyeicosatrienoic acids activate TRPV4, and 4-hydroxynonenal and $15 \mathrm{~d}-\mathrm{PGJ}_{2}$ activate TRPA1 (30-32). Our results add HODEs as endogenous ligands for TRPV1. It is noteworthy that all these TRP ligands are lipid oxidation products. It is therefore tempting to speculate that one of the major roles of certain TRP channels in mammals is to act as sensors of membrane lipid oxidation as a surrogate for cellular damage.

\section{Methods}

Animals. Adult male C56BL/6 mice (both WT and TRPV1 KO; Jackson Laboratory) and Sprague-Dawley rats (Charles River) were used for all the studies. All protocols were approved by the Institutional Animal Care and Use Committee of the University of Texas Health Science Center at San Antonio.

Drugs. All drugs were purchased from Cayman Chemicals with the exception of I-RTX (Tocris) and antibodies to 9-HODE and 13-HODE (Oxford Biomedical Research). AMG 8562 was donated by Narender Gavva (Amgen Inc.).

Preparation of skin superfusate and isolation of active component(s). Skin biopsies $(1.5 \times 1.5 \mathrm{~cm}$ from 6 mice) were dissected from the back of decapitated mice, chopped into smaller pieces, and washed for 40 minutes at room temperature $\left(22-24^{\circ} \mathrm{C}\right)$ before being exposed to modified HBSS $(2 \mathrm{mM}$ $\mathrm{CaCl}_{2}, 4 \mathrm{mM} \mathrm{NaHCO}_{3}$, and $10 \mathrm{mM}$ HEPES; $6 \mathrm{ml}$ total) at nonnoxious $\left(22-37^{\circ} \mathrm{C}\right)$ or noxious $\left(43-48^{\circ} \mathrm{C}\right)$ temperatures for 20 minutes each (each set of biopsies was only exposed to 1 elevated temperature condition). The superfusate was collected and allowed to return to room temperature $\left(22-24^{\circ} \mathrm{C}\right)$ before we carried out the calcium imaging, electrophysiological, or behavioral experiments. A similar protocol was followed in experiments evaluating the release of substances from $\mathrm{CHO}$ cells (donated by Nathaniel Jeske, University of Texas Health Science Center at San Antonio) and rat skin. In subsequent experiments, lipophilic compounds were isolated from the superfusate via C18 SepPak columns (Waters), washed with $20 \mathrm{ml}$ of $0.05 \%$ trifluoroacetic acid (v/v), and eluted with $90 \%$ acetonitrile $/ 0.05 \%$ 
A

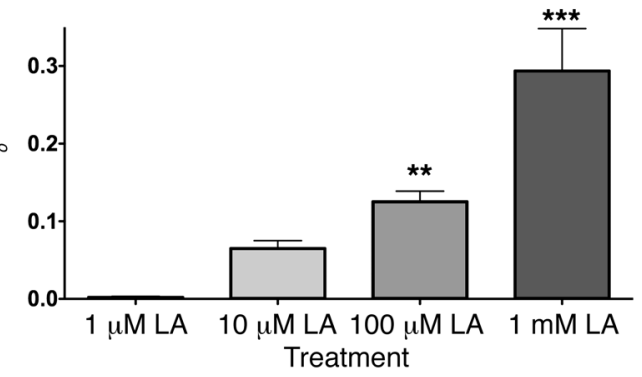

B

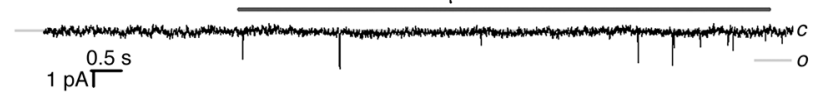

$10 \mu \mathrm{M}$ LA

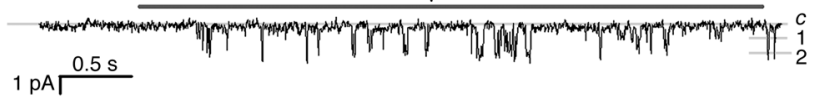

$100 \mu \mathrm{M}$ LA

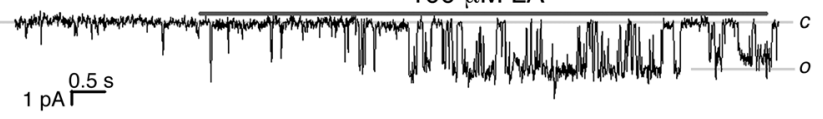

$1 \mathrm{mM} \mathrm{LA}$

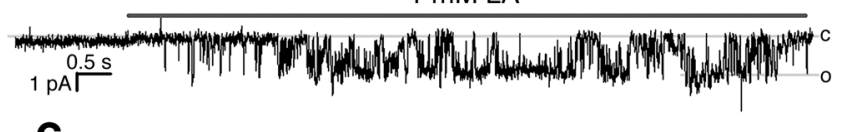

C

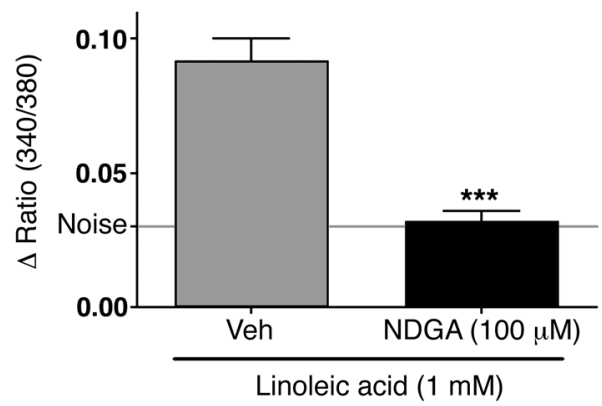

trifluoroacetic acid (TFA) (v/v). The eluate was dried under a flow of nitrogen and stored at $-80^{\circ} \mathrm{C}$ until testing, when it was redissolved in the same volume of modified Hanks buffer. When the superfusate was prepared for HPLC and MS analysis, it was eluted with $90 \%$ acetonitrile without TFA. The efficacy for activating TRPV1 was the same for freshly collected superfusate and the resuspended compounds, indicating that they were stable after collection under these conditions.

TG culturing, iCGRP release, and calcium imaging. TG culturing, iCGRP release, and calcium imaging experiments were performed as described previously (33-34) In calcium-imaging experiments, calcium accumulation ratio change below 0.03 was considered to be noise. This number was obtained after analysis of more than $10^{3}$ cells that were exposed to a flow of buffer or vehicle alone. In the calcium-imaging experiments, all drugs were applied for 1 minute. The TRPV1 mutants used in the characterization of superfusate and 9-HODE were donated by David Julius (UCSF).

Electrophysiology. The whole-cell voltage-clamp $\left(V_{b}=-60 \mathrm{mV}\right)$ recordings were performed at $22-24^{\circ} \mathrm{C}$ from the somata of TG neurons $(15-45 \mathrm{pF})$ or $\mathrm{CHO}$ cells. Single-channel recordings were carried out in cell-attached configuration as previously described (35). Data were acquired and analyzed using an Axopatch 200B amplifier and pCLAMP9.0 software (Axon Instruments). Currents were filtered with an 8-pole, low-pass Bessel filter at $0.1 \mathrm{kHz}$ (cell attached or inside out) or $0.5 \mathrm{kHz}$ (whole cell) and sampled at 1 or

\section{Figure 7}

Indirect activation of TRPV1 by linoleic acid. (A) Concentration-dependent effect of linoleic acid (LA) on $P_{o}$ of TRPV1 in transfected $\mathrm{CHO}$ cells using inside-out configuration at room temperature. Patches were washed at least 3 minutes before linoleic acid application at room temperature. (B) Representative single-channel traces demonstrating the effect of different concentrations of linoleic acid on $P_{o}$ of TRPV1expressing $\mathrm{CHO}$ cell membrane patches. $c$ is a close state. $O$ is an open state, in which the patch contained 1 channel. 1, 2, and 3 are the numbers of channels in the patches. (C) Effect of pretreatment with either vehicle or NDGA (30 $\mu \mathrm{M}, 15$ minutes) on linoleic acid-induced activation of TRPV1 transfected $\mathrm{CHO}$ cells as measured using calcium imaging $(n=25-50 ; P=0.0001) .{ }^{* \star} P<0.01 ;{ }^{* \star *} P<0.001$.

$2 \mathrm{kHz}$, respectively. Access resistance $\left(R_{s}\right)$ was compensated (40\%-80\%) when appropriate to achieve a final $R_{s}$ of $7-10 \mathrm{M} \Omega$ (whole cell). Data were rejected when $R_{s}$ changed by more than $20 \%$ during recording, leak currents were greater than $50 \mathrm{pA}$, or input resistance was less than $300 \mathrm{M} \Omega$. Currents were considered to be positive when their amplitudes were 5 -fold larger than displayed noise (in root mean square). The equations for the calculation of cell diameters and concentration-response curves were described previously. Single-channel unitary current (i) was determined from the best-fit Gaussian distribution of amplitude histograms. $P_{o}$ was calculated by normalizing $N P_{\mathrm{o}}$ for the total number of estimated channels $(N)$ in the patch. To increase accuracy in measurement of $P_{o}$, we only used patches containing 5 or fewer channels. The solutions for electrophysiological recordings were described previously $(33,36)$. Briefly, standard external solution contained $2 \mathrm{mM} \mathrm{Ca}^{2+}$. The standard pipette solution for the whole-cell configurations contained $140 \mathrm{mM} \mathrm{KCl}, 1 \mathrm{mM} \mathrm{MgCl}_{2}, 1 \mathrm{mM}$ $\mathrm{CaCl}_{2}, 10 \mathrm{mM}$ EGTA, $10 \mathrm{mM}$ D-glucose, $10 \mathrm{mM}$ HEPES, $0.2 \mathrm{mM} \mathrm{Na}$-GTP, and $2.5 \mathrm{mM} \mathrm{Mg-ATP}$ (pH 7.3). For single channel (cell attached and inside out), the bath solution consisted of $100 \mathrm{mM}$ potassium gluconate, $4 \mathrm{mM}$ $\mathrm{KCl}, 1 \mathrm{mM} \mathrm{MgCl}$, $1 \mathrm{mM}$ EGTA, $10 \mathrm{mM}$ D-glucose, and $10 \mathrm{mM}$ HEPES ( $\mathrm{pH}$ 7.3). The pipette solution contained $100 \mathrm{mM}$ sodium gluconate, $10 \mathrm{mM} \mathrm{NaCl}, 1 \mathrm{mM} \mathrm{MgCl}, 2 \mathrm{mM} \mathrm{CaCl}_{2}, 10 \mathrm{mM}$ D-glucose, and $10 \mathrm{mM}$ HEPES (pH 7.3). Drugs were applied using a fast, pressure-driven, computer-controlled 8-channel system (ValveLink8; AutoMate Scientific). For heat activation, the extracellular solution was heated by a TC-344B placed inside a SF-28 on-line heater (Warner Instruments) and locally delivered to the cells. The rate of solution flow controlled the slope of the temperature ramps. The temperature in the vicinity of the cells was recorded by a thermistor probe (Harvard Apparatus) positioned in close proximity to the cell and coupled to an Axopatch 200B amplifier and pClamp 9.0 software (Molecular Devices).

HPLC separation, bioactivity confirmation, and MS. All solvents were HPLC or optima grade and obtained from Fisher Scientific. Aliquots from control and heated skin superfusates $\left(20\right.$ minutes at $37^{\circ} \mathrm{C}$ and $48^{\circ} \mathrm{C}$, respectively) were isolated by $\mathrm{C} 18 \mathrm{Sep}$ Pak columns, dried, and dissolved initially in acetonitrile $(100 \mu \mathrm{l})$. An aliquot $(25 \mu \mathrm{l})$ was taken to dryness, dissolved in acetonitrile/water (1:9), and injected into a reversed phase HPLC column where a portion (10\%) of the TRPV1-active sample was diverted into a mass spectrometer for electrospray (negative ion) ionization. The remaining sample (90\%) for each HPLC fraction was used to assess TRPV1 biological activity using calcium imaging of $\mathrm{CHO}$ cells transfected with TRPV1. Separation of the biologically active samples from control and heated skin was carried out using a Shimadzu LC-10ADVP HPLC system and a $5 \mu \mathrm{m}$ Luna $250 \times 4.6-\mathrm{mm}$ column from Phenomenex. The flow rate was $1 \mathrm{ml} / \mathrm{min}^{-1}$ with a gradient from $10 \%$ solvent A to $90 \%$ solvent B in 30 minutes, where solvent $A$ was $8.3 \mathrm{mM}$ acetic acid, buffered to $\mathrm{pH} 5.7$ with $\mathrm{NH}_{4} \mathrm{OH}$, and solvent B was acetonitrile. Approximately $10 \%$ of the 
A

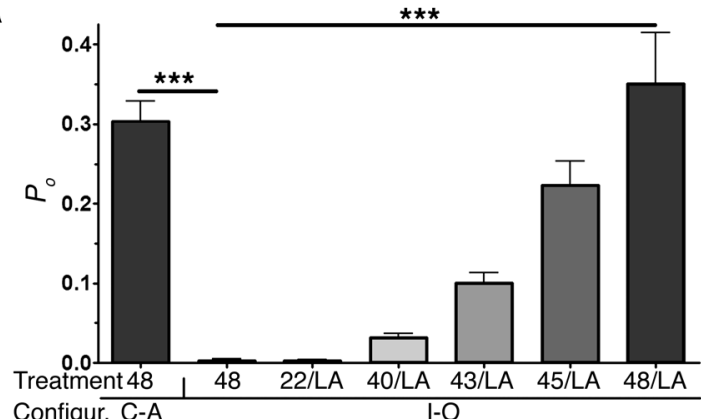

Configur. C-A

B

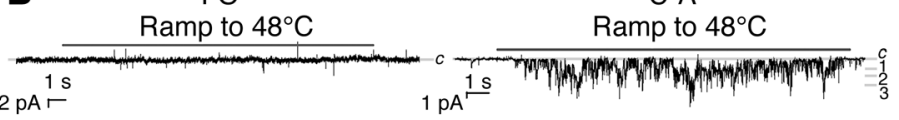

C

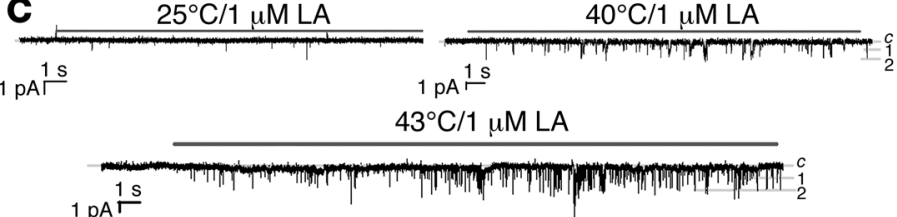

D

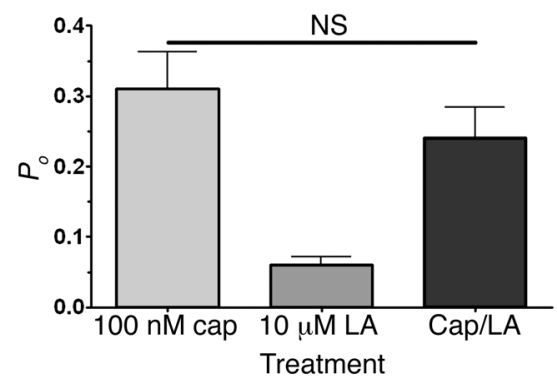

E 100 nM cap
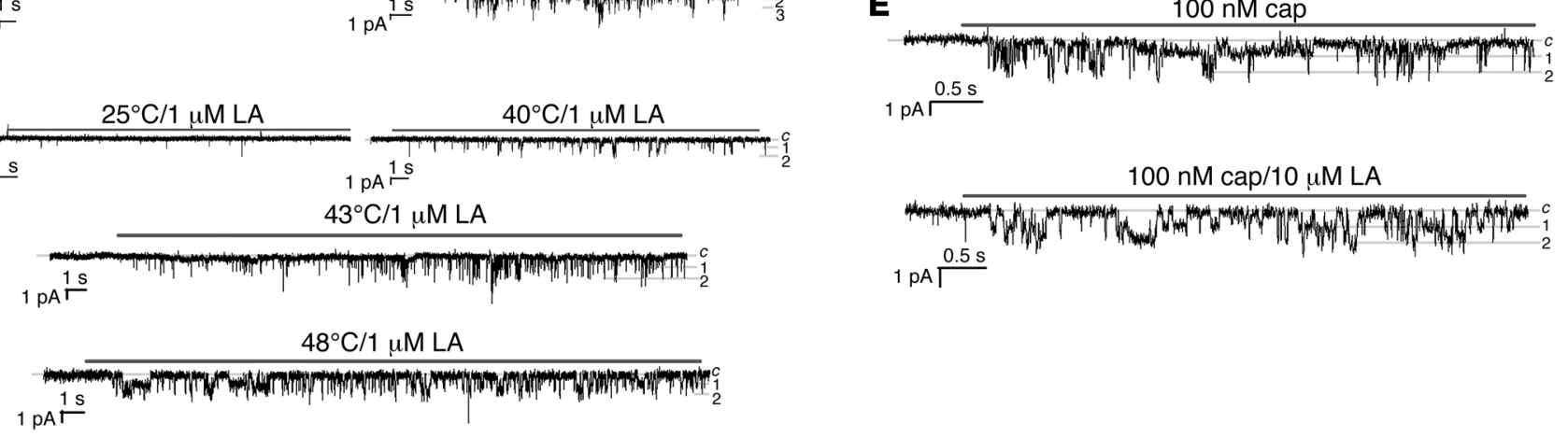

Figure 8

Linoleic acid rescues the TRPV1-mediated heat responses in membrane patches. (A) The responsiveness of TRPV1-expressing CHO cells to $48^{\circ} \mathrm{C}$ is maintained in the cell-attached configuration but is lost by 3 minutes of washing the inside-out configuration. The application of heated linoleic acid $(\mathrm{LA})(1 \mu \mathrm{M})$ rescues temperature responses (i.e., $\left.P_{0}\right)$ in inside-out patches from TRPV1-expressing cells that were washed at least 3 minutes. Single-channel configurations and applied temperatures are indicated. $(n=4-8 ; P=0.0001)$. ${ }^{* * *} P<0.001$. (B) Representative traces show single-channel recording at indicated temperatures in inside-out and cell-attached configurations. Inside-out patches were washed at least 3 minutes prior to the recording. (C) Representative traces illustrate the effects of application of $1 \mu \mathrm{M}$ LA at indicated temperatures. Recordings were performed from inside-out patches washed at least 3 minutes. (D) Coapplication of $10 \mu \mathrm{M}$ linoleic acid does not affect capsaicin-gated $(100 \mathrm{nM})$ responses in TRPV1-expressing patches. Recordings were performed from inside-out patches washed at least 3 minutes. (E) Representative traces show single-channel recording for Cap and a mix of Cap and linoleic acid.

HPLC flow was diverted into the electrospray ion source of a triple quadrupole mass spectrometer (API 3000; Applied Biosystems), and mass spectrometric analysis was performed in a negative ion mode. The remaining eluate was collected in a fraction collector and used for assessment of TRPV1 activity using calcium imaging on CHO cells expressing TRPV1. The liquid chromatography (LC)/MS dataset was then examined for the elution of specific components in the heated skin fraction compared with the control skin fraction. The biologically active fraction from a separate sample preparation (HPLC fraction 22) was analyzed by nanoelectrospray, and enhanced product ion scanning was performed on an Applied Biosystems API 4000 Q-trap, hybrid tandem quadrupole/linear ion trap mass spectrometer (Applied Biosystems). For this experiment, a sample corresponding to $25 \%$ of the entire biologically active fraction 22 from heated skin was dissolved in $200 \mu \mathrm{l}$ methanol/dichloromethane $(1: 1, \mathrm{v} / \mathrm{v})$ and placed into a NanoMate 100 (Advion Biosciences). Nanoelectrospray was initiated by applying $-1.32-\mathrm{kV}$ spray voltage and 0.15 -psi nitrogen head pressure to the sample in the pipette tip to initiate the nanospray $\left(200 \mathrm{nl} / \mathrm{min}^{-1}\right)$. The first quadrupole stage was set to pass the ion at $\mathrm{m} / \mathrm{z}$ 295 , which was collisionally activated, and product ions accumulated in the linear ion trap for enhanced product ion scanning.

Behavioral studies. Nocifensive behavior and thermal hyperalgesia were monitored by observers blinded to treatment allocation as described (37-38).
For the nocifensive behavioral assay, compounds isolated from heated skin superfusates from 12 mouse skins were redissolved in $500 \mu \mathrm{l}$ of $0.9 \%$ saline $(\mathrm{w} / \mathrm{v})$, and $50 \mu \mathrm{l}$ was injected in each hind paw.

Statistics. Data are presented as mean \pm SEM. Statistical analyses were performed using 2-tailed Student's $t$ test or 1-way ANOVA with Bonferroni's post hoc testing. A statistically significant difference was defined as $P<0.05$.

\section{Acknowledgments}

We would like to thank M. Patil, D. McMillan, and K. Hakala for excellent technical assistance. This work was supported by grants DA019585 and Clinical Translational Science Award U54RR02438 (to K.M. Hargreaves), DE017696 and DE019311 (to A.N. Akopian), and GM069338 (to R.C. Murphy).

Received for publication November 5, 2009, and accepted in revised form March 17, 2010.

Address correspondence to: Kenneth M. Hargreaves, Department of Endodontics, University of Texas Health Science Center at San Antonio, MSC 7892, 7703 Floyd Curl Dr., San Antonio, Texas 78227, USA. Phone: 210.567.3381; Fax: 210.567.3389; E-mail: hargreaves@uthscsa.edu. 
1. Brauchi S, Orta G, Salazar M, Rosenmann E, Latorre R. A hot-sensing cold receptor: C-terminal domain determines thermosensation in transient receptor potential channels. J Neurosci. 2006;26(18):4835-4840.

2. Caterina MJ, Schumacher MA, Tominaga M, Rosen TA, Levine JD, Julius D. The capsaicin receptor: a heat-activated ion channel in the pain pathway. Nature. 1997;389(6653):816-824.

3. Lumpkin EA, Caterina MJ. Mechanisms of sensory transduction in the skin. Nature. 2007;445(7130):858-865.

4. Tominaga $\mathrm{M}$, et al. The cloned capsaicin receptor integrates multiple pain-producing stimuli. Neuron 1998;21(3):531-543

5. Voets T, Droogmans G, Wissenbach U, Janssens A, Flockerzi V, Nilius B. The principle of temperaturedependent gating in cold- and heat-sensitive TRP channels. Nature. 2004;430(7001):748-754.

6. Cortright DN, Szallasi A. Biochemical pharmacology of the vanilloid receptor TRPV1. An update. Eur J Biochem. 2004;271(10):1814-1819.

7. Patwardhan AM, Scotland PE, Akopian AN, Hargreaves KM. Activation of TRPV1 in the spinal cord by oxidized linoleic acid metabolites contributes to inflammatory hyperalgesia. Proc Natl Acad SciU S A. 2009;106(44):18820-18824.

8. Caterina MJ, et al. Impaired nociception and pain sensation in mice lacking the capsaicin receptor. Science. 2000;288(5464):306-313.

9. Gavva NR, et al. The vanilloid receptor TRPV1 is tonically activated in vivo and involved in body temperature regulation. J Neurosci. 2007;27(13):3366-3374

10. Huang SM, et al. Overexpressed transient receptor potential vanilloid 3 ion channels in skin keratinocytes modulate pain sensitivity via prostaglandin E2. J Neurosci. 2008;28(51):13727-13737.

11. Lopshire JC, Nicol GD. The cAMP transduction cascade mediates the prostaglandin E2 enhancement of the capsaicin-elicited current in rat sensory neurons: whole-cell and single-channel studies. J Neurosci. 1998;18(16):6081-6092.

12. Zhang X, Huang J, McNaughton PA. NGF rapidly increases membrane expression of TRPV1 heat-gated ion channels. EMBOJ. 2005;24(24):4211-4223.

13. Wheelan P, Zirrolli JA, Murphy RC. Low-energy fast atom bombardment tandem mass spectrometry of monohydroxy substituted unsaturated fatty acids. Biol Mass Spectrom. 1993;22(8):465-473.

14. van Heuven-Nolsen D, Muis T, Engels F, Henricks PA, Buckley TL, Nijkamp FP. Hypoten- sive effect of 13-hydroxylinoleic acid in the rat: mediation via the release of a CGRP-like mediator from capsaicin-sensitive nerves. $\mathrm{Br} J$ Pharmacol. 1995;115(5):835-839.

15. Yoshida Y, Niki E. Bio-markers of lipid peroxidation in vivo: hydroxyoctadecadienoic acid and hydroxycholesterol. Biofactors. 2006;27(1-4):195-202.

16. Yoshida Y, et al. Hydroxyoctadecadienoic acid and oxidatively modified peroxiredoxins in the blood of Alzheimer's disease patients and their potential as biomarkers. Neurobiol Aging. 2009;30(2):174-185.

17. Cesare P, McNaughton P. A novel heat-activated current in nociceptive neurons and its sensitization by bradykinin. Proc Natl Acad Sci U S A. 1996;93(26):15435-15439.

18. Jordt SE, Julius D. Molecular basis for speciesspecific sensitivity to "hot" chili peppers. Cell. 2002;108(3):421-430

19. Lehto SG, et al. Antihyperalgesic effects of (R,E)$\mathrm{N}$-(2-hydroxy-2,3-dihydro-1H-inden-4-yl)-3-(2 (piperidin-1-yl)-4-(tri fluoromethyl)phenyl)-acrylamide (AMG8562), a novel transient receptor potential vanilloid type 1 modulator that does not cause hyperthermia in rats. J Pharmacol Exp Ther. 2008;326(1):218-229.

20. Dupont R, et al. New bis-catechols 5-lipoxygenase inhibitors. Bioorg Med Chem. 2001;9(2):229-235

21. Goodman Y, Steiner MR, Steiner SM, Mattson MP. Nordihydroguaiaretic acid protects hippocampal neurons against amyloid beta-peptide toxicity, and attenuates free radical and calcium accumulation. Brain Res. 1994;654(1):171-176.

22. Camp RD, Mallet AI, Woollard PM, Brain SD, Black AK, Greaves MW. The identification of hydroxy fatty acids in psoriatic skin. Prostaglandins. 1983;26(3):431-447.

23. Jira W, Spiteller G, Richter A. Increased levels of lipid oxidation products in low density lipoproteins of patients suffering from rheumatoid arthritis. Chem Phys Lipids. 1997;87(1):81-89.

24. Watanabe H, Vriens J, Suh SH, Benham CD, Droogmans G, Nilius B. Heat-evoked activation of TRPV4 channels in a HEK293 cell expression system and in native mouse aorta endothelial cells. J Biol Chem. 2002;277(49):47044-47051

25 . Ben-Shabat $S$, et al. An entourage effect: inactive endogenous fatty acid glycerol esters enhance 2 arachidonoyl-glycerol cannabinoid activity. Eur J Pharmacol. 1998;353(1):23-31.

26. De Petrocellis L, Chu CJ, Moriello AS, Kellner JC, Walker JM, Di Marzo V. Actions of two naturally occurring saturated $\mathrm{N}$-acyldopamines on transient receptor potential vanilloid 1 (TRPV1) channels. $\mathrm{Br}$ JPharmacol. 2004:143(2):251-256.

27. Yoshida Y, et al. Hydroxyoctadecadienoic acid and oxidatively modified peroxiredoxins in the blood of Alzheimer's disease patients and their potential as biomarkers. Neurobiol Aging. 2009;30(2):174-185.

28. Chinetti G, Fruchart JC, Staels B. Peroxisome proliferator-activated receptors (PPARs): nuclear receptors at the crossroads between lipid metabolism and inflammation. Inflamm Res. 2000;49(10):497-505.

29 . Green FA. Generation and metabolism of lipoxygenase products in normal and membrane-damaged cultured human keratinocytes. J Invest Dermatol. 1989;93(4):486-491.

30. Andersson DA, Gentry C, Moss S, Bevan S. Transient receptor potential A1 is a sensory receptor for multiple products of oxidative stress. J Neurosci. 2008;28(10):2485-2494.

31. Trevisani M, et al. 4-Hydroxynonenal, an endogenous aldehyde, causes pain and neurogenic inflammation through activation of the irritant receptor TRPA1. Proc Natl Acad Sci U S A. 2007;104(33):13519-13524.

32. Watanabe H, Vriens J, Prenen J, Droogmans G, Voets T, Nilius B. Anandamide and arachidonic acid use epoxyeicosatrienoic acids to activate TRPV4 channels. Nature. 2003;424(6947):434-438.

33. Akopian AN, Ruparel NB, Jeske NA, Hargreaves KM. Transient receptor potential TRPA1 channel desensitization in sensory neurons is agonist dependent and regulated by TRPV1-directed internalization. J Physiol. 2007;583(Pt 1):175-193.

34. Patwardhan AM, et al. PAR-2 agonists activate trigeminal nociceptors and induce functional competence in the delta opioid receptor. Pain. 2006;125(1-2):114-124.

35. Premkumar LS, Agarwal S, Steffen D. Single-channel properties of native and cloned rat vanilloid receptors. J Physiol. 2002;545(Pt 1):107-117.

36. Akopian AN, Ruparel NB, Patwardhan A, Hargreaves KM. Cannabinoids desensitize capsaicin and mustard oil responses in sensory neurons via TRPA1 activation. J Neurosci. 2008;28(5):1064-1075.

37. Hargreaves K, Dubner R, Brown F, Flores C, Joris $\mathrm{J}$. A new and sensitive method for measuring thermal nociception in cutaneous hyperalgesia. Pain. 1988;32(1):77-88.

38. Ruparel NB, Patwardhan AM, Akopian AN, Hargreaves KM. Homologous and heterologous desensitization of capsaicin and mustard oil responses utilize different cellular pathways in nociceptors. Pain. 2008;135(3):271-279. 